Yu Sun

Tiecheng $W u$

Yumin Su

Huanghua Peng

http://dx.doi.org/10.21278/brod71401

\title{
NUMERICAL PREDICTION ON VIBRATION AND NOISE REDUCTION EFFECTS OF PROPELLER BOSS CAP FINS ON A PROPULSION SYSTEM
}

UDC 629.5.035

Original scientific paper

\begin{abstract}
Summary
To investigate the vibration and noise reduction effects of Propeller Boss Cap Fins (PBCF), the Large Eddy Simulation (LES) method has been employed in the noise performance estimation of a propeller-rudder system. The hydrodynamic performance of the propulsion system is predicted after the grid independence analysis, then further compared with the result of cavitation tunnel experiment. The acoustic simulation is performed based on Ffowcs Williams and Hawkings (FW-H) equation. After the observation of the hydrodynamic noise performance changes, the forces of propulsion systems and noise reduction effects of PBCF are analyzed. It's indicated from the research results that PBCF can not only improve the propulsion efficiency, but also reduce the radiation noise intensity significantly. Meanwhile, the lateral force fluctuation of hub cap can be decreased by suppressing the vibration of propeller shaft. In addition, the time-averaged value of the rudder lateral force has been decreased by about $15.5 \%$. It has been well known that the radiation of propulsion noise is directional. Accordingly, it is found that the noise reduction effects due to PBCF are also directional, which is the most noticeable in the axial direction.
\end{abstract}

Key words: $\quad$ Large eddy simulation; Propeller-rudder system; Propeller boss cap fin; Noise reduction; Acoustic simulation

\section{Introduction}

Plenty of ship Energy Saving Devices (ESDs) can reduce the propulsion vibration and noise. In 1988, Ouchi first introduced the Propeller Boss Cap Fins (PBCF) to recovery the rotational energy from the hub vortex and increase the propulsive efficiency. PBCF can produce a torque behind the propeller blades, which offsets a part of the propeller torque. The hub vortex intensity is reduced, and the cavitation performance is improved [1,2].

There are some earlier researches about PBCF. Ouchi and Tamashima [3] carried out some systematic investigations on PBCF. The results showed that PBCF was an excellent ESD which could reduce the hub vortex and increase the propulsive efficiency with a minor improvement. Then Ouchi et al. [4] carried out an experimental research on the noise reduction effect of PBCF under the cavitation pattern. PBCF could reduce the propulsive Sound Pressure 
Yu Sun, Tiecheng Wu,

Yumin Su, Huanghua Peng
Numerical Prediction on Vibration and Noise Reduction Effects of Propeller Boss Cap Fins on a Propulsion System

Level (SPL) by 3 6 dB. To observe the velocity distributions behind the hub, Particle Image Velocimetry (PIV) was applied in the tests by Dang [5]. It was indicated that the vortex was reduced significantly. Hans et al. [6] conducted a series of full-scale trials on an Aframax tanker. The results confirmed the energy saving effects of PBCF. Kawamura et al. [7] also carried out the model test and the real ship test at the same time, and compared the energy saving effect of PBCF at different scales. It was found that the PBCF installed on the real ship had higher energy saving effect. Kawamura considered that the scale effect, interference of appendages, roughness of blade surface and propeller cavitation led to this phenomenon. In addition to the experimental testing method, numerical simulation methods (Computational Fluid Dynamics-CFD and Surface Panel Method-SPM) are also important methods for propeller hydrodynamic performance research, Vlašić et al. [8] performed the numerical simulations based on potential and viscous flow theory for five Gawn series propellers. Lee et al. [9] and Jang et al. [10] applied CFD method to assess the performance of a partially submerged propeller under the bollard condition, and sinusoidal pitch motion conditions in several conditions of varying pitch angle. Ghassemi et al. [11] with his team used SPM method to analyze the influence of the PBCF parameters on the propulsive efficiency. The influence of the hub radial ratio, installation location and attack angle on the propulsive performance was studied. Xiong et al. [12] applied the CFD method to the simulation of the effect of PBCF on propulsion performance. After the gradual parameter optimizations, the PBCF with the best energy saving effect was found. A PBCF design method by Ma et al. [13] could improve the propulsive efficiency effectively. The experimental data verified the reliability of his method. Mizzi et al. [14] demonstrated an approach for optimising PBCF by using CFD analysis, it was indicated that the efficiency was increased by $1.3 \%$ due to the hub vortex weakened. Gaggero [15] also identified a design strategy and the efficiency increase could reach $4 \%$ after his optimization. The current researches on PBCF are very few which mainly focus on their hydrodynamic performance improvement and neglect the other effects. Therefore, Research emphasis of this paper is the vibration and noise reduction effects of PBCF. Wang et al. [16, 17] applied CFD method to the prediction the hydrodynamic performance of some propellers in cavitating flow and observed the flow wake distributions during the heave and oscillating motions. Then Wang et al. [18] analyzed the vortex of a submarine propeller operating near the surface, the comparison between the experimental and numerical results showed the vortex simulation reliability. In the study of Bagheri et al. [19], the hydrodynamics and noise prediction of a five blade marine propeller were analyzed through numerical and experimental methods under cavitating and non-cavitating conditions. Aktas et al. [20] and Wu et al. [21] both presented some advanced joint time-frequency analysis procedures to study propeller cavitation-induced noise. The results showed that the cavitation had an impact on the noise performance. Ebrahimi et al. [22] investigated the noise performances of ducted propellers in a cavitation tunnel. The impact of the parameters including the number of blades, diameter, expanded area ratio, pitch, rake and skew were discussed in their research. Cianferra et al. [23] applied CFD method to the noise performance prediction. Different noise generation mechanisms were investigated separately in his work. In the previous researches, the energy-saving effects due to PBCF were studied, while the influences of rudders on the hydrodynamic performance of the propulsion system were ignored. This paper presents the numerical investigation on the vibration and noise reduction effect of PBCF on a propulsion system close to a real working environment. Therefore, the conclusion of this paper is more reasonable.

In this paper, the technical skills to save energy and reduce the noise of PBCF have been investigated. Large Eddy Simulation (LES) method is applied to noise reduction effect analysis, and the numerical method is introduced. In addition, the cavitation tunnel experiment has been also performed. Based on the comparison of the calculational and experimental data, grid independence and result reliability are verified. Then the noise performance and fluctuating 
force are observed with and without PBCF, and the influence on the whole propulsion system is analyzed according to the change of the flow field. Finally, the noise and vibration reduction mechanism of PBCF has been studied.

\section{Theories and Formulas}

\subsection{Turbulence model}

Motion-compliant continuity and momentum conservation equations of the LES method for incompressible Newtonian fluid motion are as follows.

$$
\begin{gathered}
\frac{\partial \bar{u}_{i}}{\partial x_{i}}=0 \\
\frac{\partial \bar{u}_{i}}{\partial t}+\frac{\partial}{\partial x_{j}}\left(\overline{u_{i} \bar{u}_{j}}\right)=-\frac{1}{\rho} \frac{\partial \bar{p}}{\partial x_{i}}+v \frac{\partial^{2} \bar{u}_{i}}{\partial x_{j}^{2}}-\frac{\partial}{\partial x_{j}} \tau_{i j}^{S}
\end{gathered}
$$

where $u_{i}$ and $u_{j}$ are the time-average values $(i, j=1,2,3)$ of the velocity component, $p$ is the time-average pressure, the overline represents the physical quantity after filtering, $\rho$ is the fluid density which is a fixed value, $v$ is the kinematical viscosity coefficient, and $\tau_{i j}^{S}=\overline{u_{i}} \bar{u}_{j}-\overline{u_{i} u_{j}}$ is the Subgrid Reynolds Stress (SGS) $[24,25,26]$.

This study applied LES method to predict the hydrodynamic performance. The boxy filtering function was employed in all the simulations. Firstly, SST $K-\omega$ model was applied to the steady performance simulation until the iteration convergence. Then the turbulence model was replaced as LES with the Smargorinsky-Lily mode, and the unsteady force and flow field were simulated $[27,28,29]$. In all the simulations which were run by using the Pressure-Based solver [30, 31], the SIMPLEC algorithm was applied for Pressure-Velocity Coupling, with Least Squares Cell Based for gradient discretization, with PRESTO for pressure discretization, and with Bounded Central Differencing for momentum discretization. Sliding mesh technique was applied to the rotation motion simulation. To observe the noise performance, the time step was set as $10^{-5} \mathrm{~s}$ and 20 iterations were proceeded in each step [32, 33].

\subsection{Hydrodynamic performance coefficients}

The hydrodynamic coefficients of the propulsion system were calculated according to the following equations.

$$
K_{t}=\frac{T_{p}}{\rho n^{2} D^{4}}, K_{a}=\frac{T_{p}+T_{R}}{\rho n^{2} D^{4}}, K_{q}=\frac{Q}{\rho n^{2} D^{5}}, J=\frac{V_{\mathrm{a}}}{n D}, \eta_{a}=\frac{K_{a}}{K_{q}} \cdot \frac{J}{2 \pi}
$$

where $K_{a}, K_{t}$ and $K_{r}$ are the thrust coefficients of entire propulsion system, propeller with or without PBCF and rudder, $K_{q}$ is the torque coefficient of propeller, $\eta$ and $\eta_{a}$ are the efficiencies of propulsion systems without rudder and with rudder, $T_{P}$ and $T_{R}$ represent the thrust generated by propeller with or without PBCF and rudder, $Q$ is the torque of propeller, $\rho$ is the fluid density, $V_{\mathrm{a}}$ is the flow speed in the tunnel, $n$ is propeller rotational speed, $D$ is the diameter of propeller disk, and $J$ is the advance coefficient. The fluid in the tunnel is incompressible, and $\rho$ is a fixed value.

\subsection{Acoustic model}

After completion of the flow field calculation, Ffowcs Williams and Hawkings (FW-H) equation based on LES is added into the acoustic simulation [33, 34], of which the reference 
Yu Sun, Tiecheng Wu, Yumin Su, Huanghua Peng
Numerical Prediction on Vibration and Noise Reduction Effects of Propeller Boss Cap Fins on a Propulsion System

sound pressure is $10^{-6} \mathrm{~Pa}$ and the sound speed is $1500 \mathrm{~m} / \mathrm{s}$ in the water liquid. The equation is as follows.

$$
\begin{aligned}
\frac{1}{c_{0}} \frac{\partial^{2} p^{\prime}}{\partial t^{2}}-\nabla^{2} p^{\prime}= & \frac{\partial}{\partial t}\left\{\left[\rho_{0} v_{n}+\rho\left(u_{n}-v_{n}\right)\right] \delta(f)\right\} \\
& -\frac{\partial}{\partial x_{i}}\left\{\left[P_{i j} n_{j}+\rho u_{i}\left(u_{n}-v_{n}\right)\right] \delta(f)\right\}-\frac{\partial^{2}}{\partial x_{i} \partial x_{j}}\left[T_{i j} H(f)\right] \\
T_{i j}= & \rho u_{i} u_{i}+\delta_{i j}\left(p^{\prime}-c_{0}^{2}\left(\rho-\rho_{0}\right)\right)-\tau_{i j},
\end{aligned}
$$

Where $c_{0}$ and $\rho_{0}$ are the reference sound speed and medium density, $\rho$ is fluid density under turbulence, $p^{\prime}=p-p_{0}$ is sound pressure of disturbed flow field, $\delta_{i j}$ represents Kronecker product function, $\delta(f)$ and $H(f)$ represent Dirac delta function and Heaviside function, $f$ represents wall function, $u_{n}$ stands for flow velocity component in $x_{i}$ direction, $v_{n}$ stands for normal velocity component on the wall, and $T_{i j}$ is the Lighthill stress tensor.

In addition, SPL distance attenuation formula is as follows [35].

$$
L=L_{0}-20 \lg \left(r / r_{0}\right)
$$

where $L_{0}$ and $r_{0}$ are the total SPL and the sound source distance of the known position respectively, $L$ and $r$ are the total SPL and the sound source distance of the calculation position.

\section{Experimental setup and its numerical modelling}

\subsection{Introduction to cavitation tunnel}

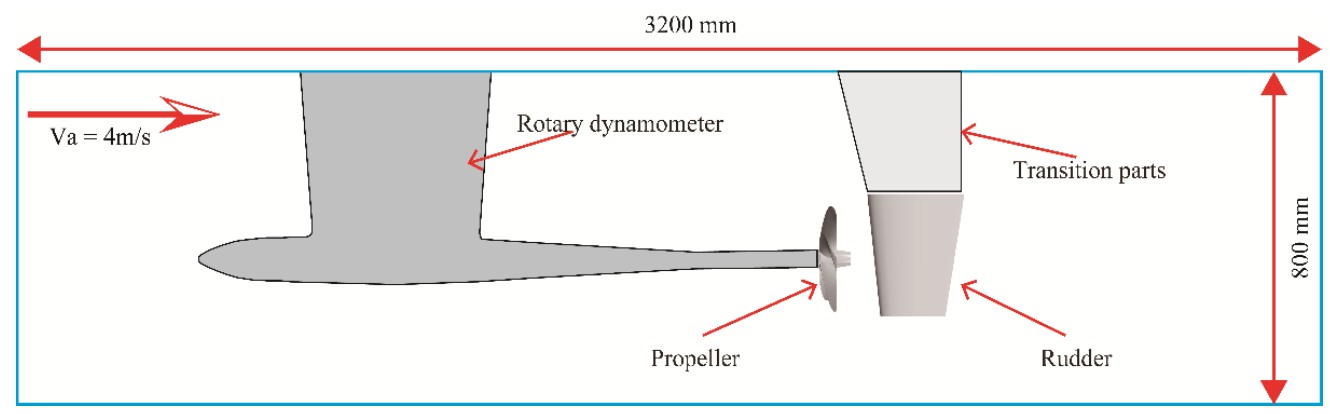

(a) Experiment diagrammatic sketch.

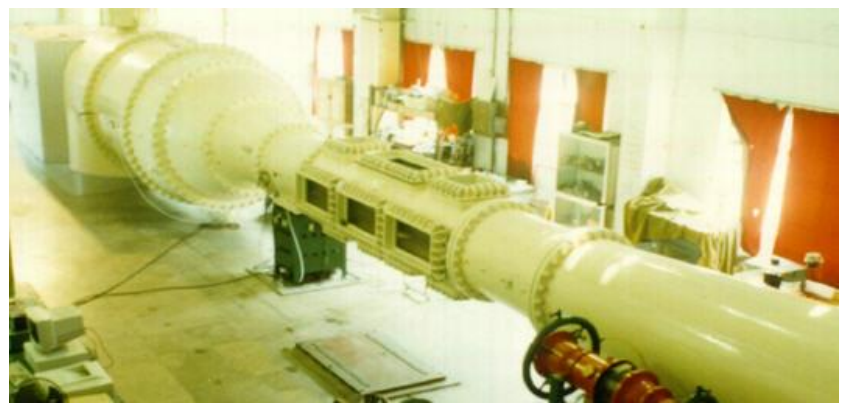

(b) Cavitation tunnel.

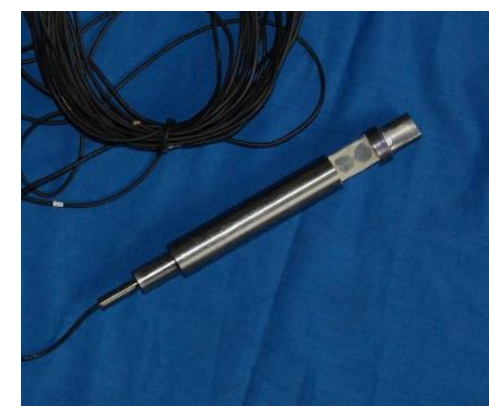

(c) Force balance.

Fig. 1 Cavitation tunnel experiment.

Fig.1 introduces the equipment and details of the experimental scheme. All the tests are performed in the cavitation tunnel. The cylindrical working section is $3.2 \mathrm{~m}$ long which ensures the propeller wake fully developed, and its diameter is 3.2 times wider than the propeller disk. 
Hence there is no blockage effect to be worried. The flow velocity range in the tunnel is from $3 \mathrm{~m} / \mathrm{s}$ to $20 \mathrm{~m} / \mathrm{s}$, and the turbulence intensity in present tunnel is less than $2 \%$. The propulsive thrust and torque are measured by the rotary dynamometer, and the rudder force is obtained by the force balance showed in Fig.1 (c).

\subsection{Major model parameters and test conditions}

A propulsion system model of a certain container ship is shown in Fig.2. The system includes a 5-blade propeller, a 5-blade PBCF and a rudder. The diameter of the propeller and $\mathrm{PBCF}$ is $250 \mathrm{~mm}$ and $70 \mathrm{~mm}$, respectively. The pitch ratio of the propeller is 0.9510 . The section of the rudder is NACA0018, and the distance between the rudder shaft and the hub end is $140 \mathrm{~mm}$.

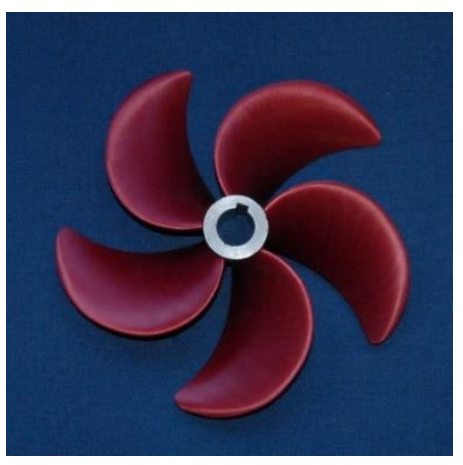

(a)

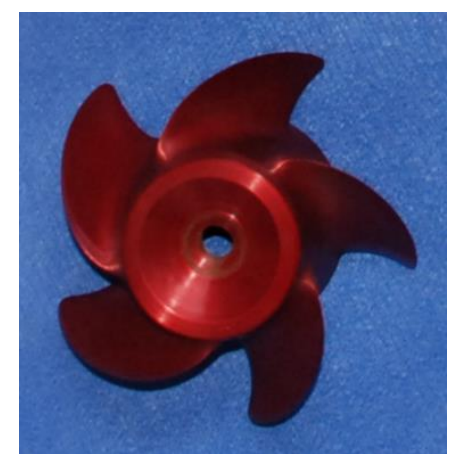

(b)

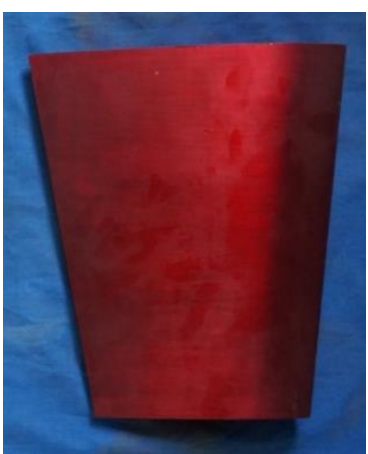

(c)

Fig. 2 Experimental models of propulsion system. (a) Propeller; (b) PBCF; (c) Rudder.

Experimental pictures are given in Fig.3. The propulsion system without PBCF was installed. The hydrodynamic forces were measured, which included propulsive thrust and torque. The force on the rudder was obtained separately by a force balance. Under atmospheric pressure, the advance coefficient $J$ of the propulsion ranged from 0.45 to 1.00 , which increased 0.05 in each test. The flow speed $V_{\mathrm{a}}$ in the tunnel was fixed $4 \mathrm{~m} / \mathrm{s}$. $J$ was changed by the propeller rotational speed increase. After all the above tests finished, the hub cap was replaced by PBCF and the former measurements were repeated exactly.

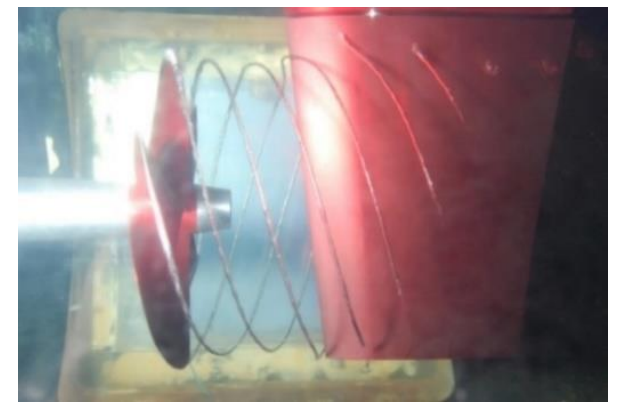

(a)

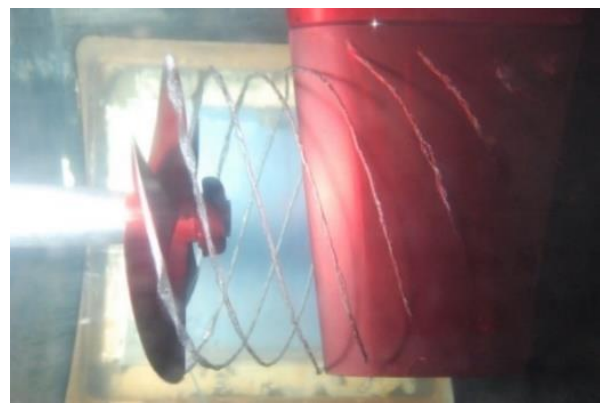

(b)

Fig. 3 Experimental pictures of propulsion systems. (a) Propeller + Rudder; (b) Propeller +PBCF + Rudder.

\subsection{Numerical modelling}

The hydrodynamic performance predictions were proceeded by applying the FLUENT (Version 19.2) solution technique. The establishment process of the numerical model is described as below.

The computational flow field of the propulsion was divided into three parts by two cylinders, and the propeller disk center was placed at the coordinate origin. The division of domains is shown in Fig.4a. Domain 1 and Domain 2 were where the propeller and rudder are 
Yu Sun, Tiecheng Wu, Yumin Su, Huanghua Peng
Numerical Prediction on Vibration and Noise Reduction Effects of Propeller Boss Cap Fins on a Propulsion System

located. The grids in the two domains were unstructured tetrahedral ones and the grids of thin edges were refined. Domain 3 involved the rest of the flow field and was filled with structured hexahedral grids. The diameter of the cylinder field was 3.2 times propeller disk diameter $D$. The distances from the propeller disk to the inlet and the outlet were $3.2 D$ and $8 D$, separately. Therefore, the flow field could be fully developed. The fluid velocity $V_{\mathrm{a}}$ and the advance coefficient $J$ were set according to the experimental scheme.

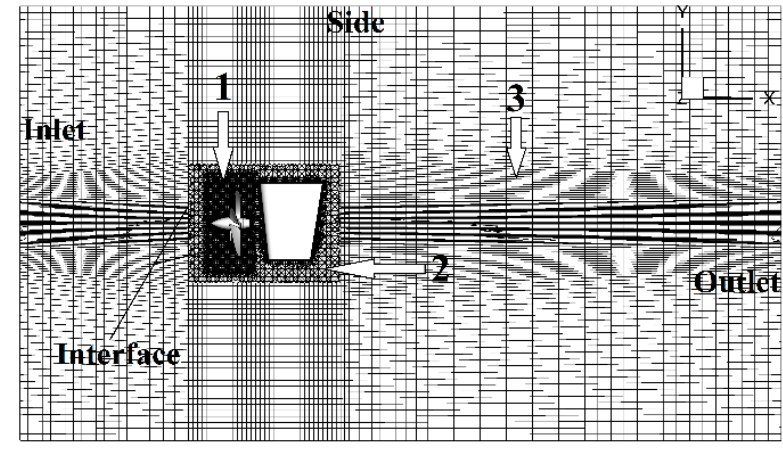

(a)

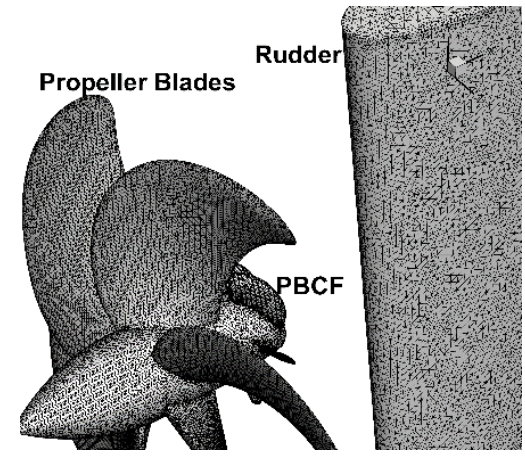

(b)

Fig. 4 Mesh of calculation model (Medium grids). (a) Computational domain; (b) Surface mesh.

At high Reynolds numbers, layer meshes are needed for turbulence prediction [36, 37]. Therefore, adaptive grids with prismatic layer mesh are generated in this work. To make sure $y^{+}$values less than 1 , the thickness of the layer mesh stick to the model was $10^{-5} \mathrm{D}$ with a stretching factor of 1.10. The coarse, medium and fine meshing schemes were created to validate the grid independence. The grid numbers of the three schemes were 4.95 million, 8.23 million and 13.01 million, respectively. The medium meshing scheme is shown in Fig.4. The results of three meshing schemes given in Table 1 coincide reasonably well, so the increased mesh density had little impact on the propulsive efficiency results. Therefore, the scheme of the medium grid was applied to the hydrodynamic predictions.

Table 1 Grid independence validation results.

\begin{tabular}{ccccc}
\hline \hline$J$ & Hydrodynamic coefficients & Coarse grids & Medium grids & Fine grids \\
\hline \multirow{3}{*}{0.7} & $K_{\mathrm{a}}$ & 0.192 & 0.191 & 0.191 \\
& $10 K_{\mathrm{Q}}$ & 0.326 & 0.326 & 0.325 \\
& $\eta_{a}$ & 0.656 & 0.654 & 0.653 \\
\hline \multirow{3}{*}{0.8} & $K_{\mathrm{a}}$ & 0.141 & 0.140 & 0.140 \\
& $10 K_{\mathrm{Q}}$ & 0.265 & 0.264 & 0.264 \\
& $\eta_{a}$ & 0.677 & 0.676 & 0.676 \\
\hline \multirow{3}{*}{0.9} & $K_{\mathrm{a}}$ & 0.086 & 0.085 & 0.085 \\
& $10 K_{\mathrm{Q}}$ & 0.195 & 0.194 & 0.193 \\
& $\eta_{a}$ & 0.629 & 0.628 & 0.627 \\
\hline
\end{tabular}

\section{Result and discussion}

\subsection{Validation of acoustic model}

According to the FW-H equation, the acoustic simulation is carried out on the basis of flow field distribution [38]. The investigation on the flow field of NACA airfoil are very systematic. Therefore, the hydrodynamic noise performance is verified by the NACA0018 airfoil simulation [32]. 


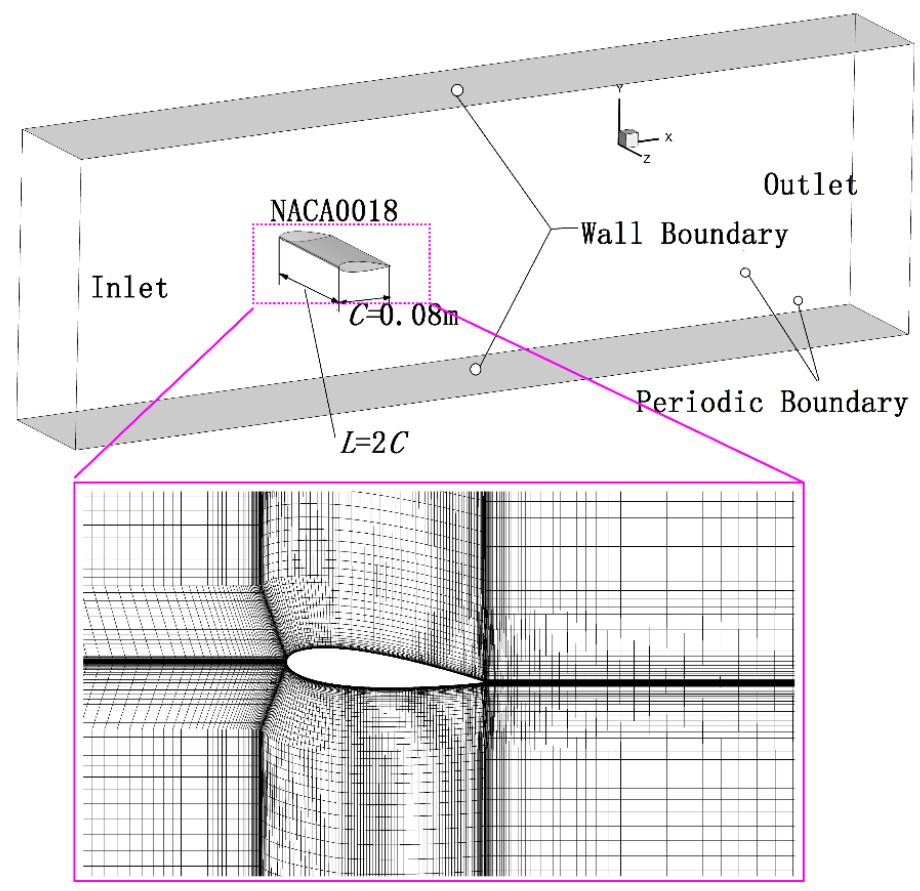

Fig. 5 Cavitation tunnel experiment.

According to the computational model [32], the domain and mesh were established. The airfoil chord length was $C(0.08 \mathrm{~m})$, and its span length was $2 C$. To make sure the flow field fully developed, the distance between the inlet and the leading edge was $5 C$, the distance between the inlet and the leading edge was $10 C$, the distance between the upper and lower sides was $5 C$, and the distance between the right and left sides was as much as the span length. The settings of boundary conditions are shown in Fig.5. The inlet was set as the velocity inlet, and the velocity of flow field was $30 \mathrm{~m} / \mathrm{s}$. The outlet was set as pressure outlet, the right and left sides were set as the periodic boundary, and the rest surfaces and boundaries were set as the wall. At this time, the Reynolds number was $1.6 \times 10^{5}$. To keep the $y^{+}$under 1 , the thickness of the first prismatic layer mesh was set to $10^{-5} \mathrm{~m}$ with a stretching factor of 1.10 . The reference sound pressure is $2 \times 10^{-5} \mathrm{~Pa}$ and the sound speed is $340 \mathrm{~m} / \mathrm{s}$ in the air. The rest settings were as same as the calculation model of the propulsion system. After the simulations, the pressure distributions can be obtained. According to Equation 6, the pressure coefficient $C_{p}$ along the chord direction is calculated and compared with the reference result.

$$
C_{p}=\frac{P}{\frac{1}{2} \rho_{\text {air }} V^{2}}
$$

where $P$ is the pressure on the airfoil surface, $\rho_{\text {air }}$ is the air density, $V$ is the flow speed.

Table 2 Position comparison of flow separation.

\begin{tabular}{ccccc}
\hline \hline $\begin{array}{c}\text { Attack } \\
\text { angle }\left({ }^{\circ}\right)\end{array}$ & NACA0018 & $\begin{array}{c}\text { Experimental } \\
\text { result }(X / C)\end{array}$ & $\begin{array}{c}\text { Reference } \\
\text { result }(X / C)\end{array}$ & $\begin{array}{c}\text { Calculation } \\
\text { result }(X / C)\end{array}$ \\
\hline \multirow{2}{*}{3} & Pressure side & 0.611 & 0.66 & 0.63 \\
& Suction side & 0.374 & 0.42 & 0.37 \\
\hline \multirow{2}{*}{6} & Pressure side & 0.707 & 0.76 & 0.72 \\
& Suction side & 0.204 & 0.29 & 0.25 \\
\hline
\end{tabular}


Yu Sun, Tiecheng Wu, Yumin Su, Huanghua Peng

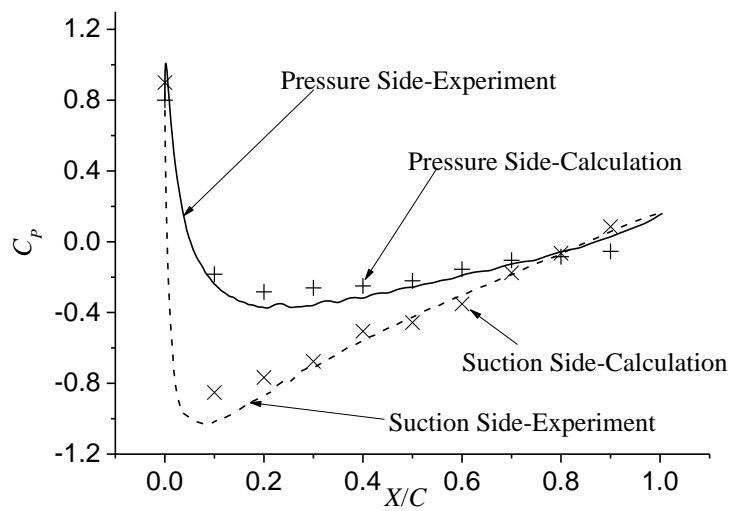

(a)
Numerical Prediction on Vibration and Noise Reduction Effects of Propeller Boss Cap Fins on a Propulsion System

Fig. 6 Pressure distribution of NACA0018 airfoil. (a) $\alpha=3^{\circ} ;$ (b) $\alpha=6^{\circ}$.

The pressure distribution and the flow separation position at attack angles of $3^{\circ}$ and $6^{\circ}$ are given in Fig. 6 and Table 2. According to the contrast result, the flow field prediction is considered reliable.

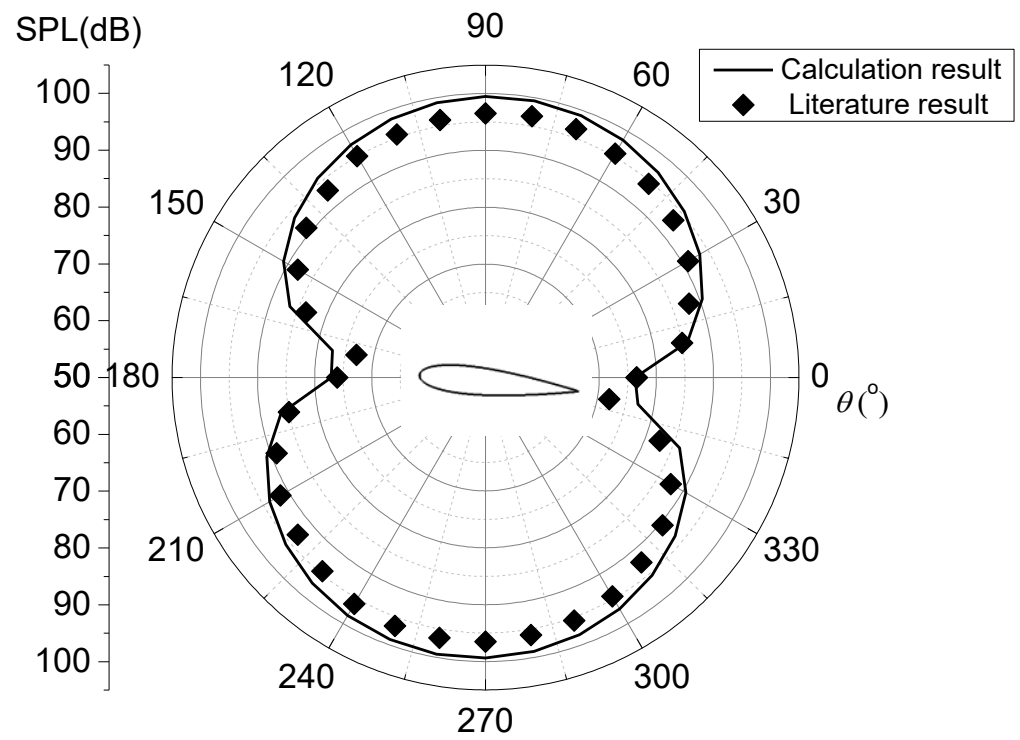

Fig. 7 Total Sound Pressure Level (SPL) of NACA0018 airfoil $\left(\alpha=6^{\circ}\right)$.

Depending on the way of noise performance calculation [32], the SPL 10m away from the airfoil was calculated. Then the data was transformed to the SPL $0.095 \mathrm{~m}$ away from the airfoil by noise attenuation formula. The contrast result is given in Fig.7. It is indicated that the radiated noise intensity of NACA0018 airfoil is directional, of which the SPL in the chord direction is the smallest, and the SPL perpendicular to the chord direction is the largest. The total SPLs at different angles are in the shape of " 8 ". The calculation result of sound field distribution agrees well with that in the literature. Meanwhile, the error is within the acceptable range. Therefore, the reliability of the noise prediction method is verified.

\subsection{Hydrodynamic performance coefficients}

The calculated and experimental values of $K_{a}, K_{Q}$ and $\eta_{a}$ are plotted and compared. The results are presented in Fig.8. In the following figures, the suffixes "EFD" and "CFD" stand for experimental values and calculated values separately, " $A$ " and " $B$ " represent the propellerrudder system and the propeller-PBCF-rudder system. According to the comparison results, the error of hydrodynamic performance coefficients at $J=0.8$ is within $3 \%$, and the calculation models of this work are considered reliable. The calculation results indicate that the propulsion 
efficiency with PBCF is higher. The energy saving effect obtained in the experiment is $1.47 \%$ at the design advance coefficient $J=0.8$. Due to the difference between the experimental model and simulation model, the hydrodynamic performance error can't be avoided, but the energy saving effect result of the simulation is coincided with the experiment result. Therefore, the simulation results are believed reliable.

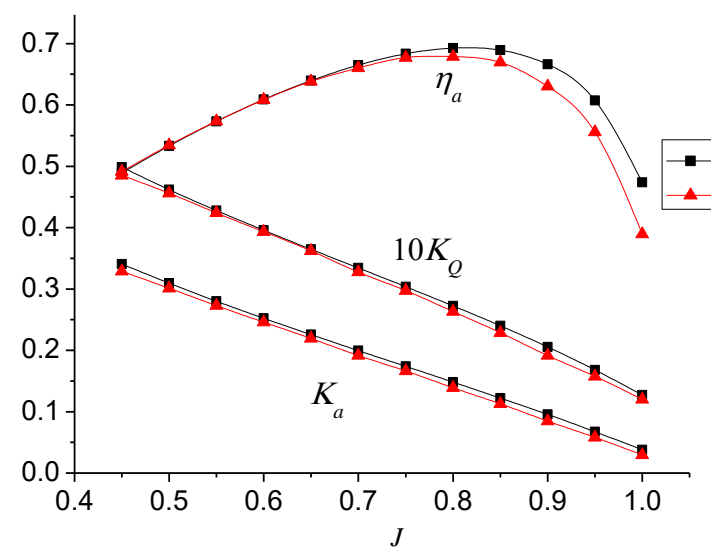

(a) Propeller + Rudder

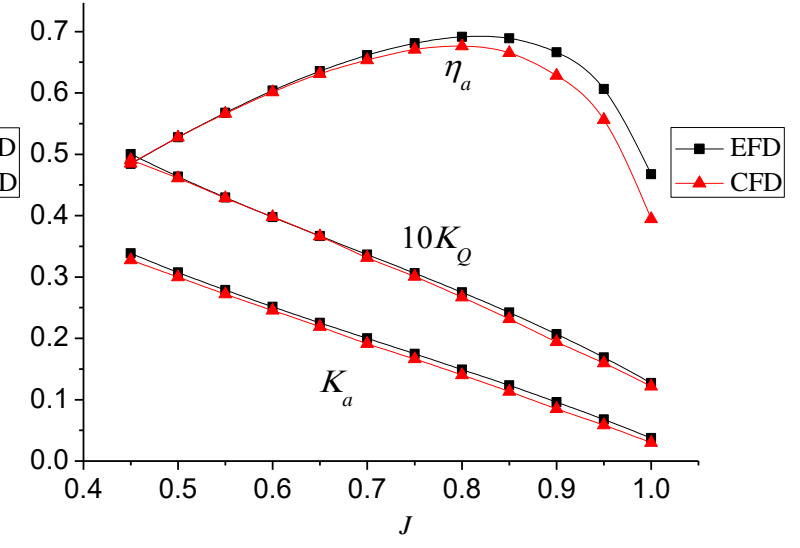

(b) Propeller + PBCF + Rudder.

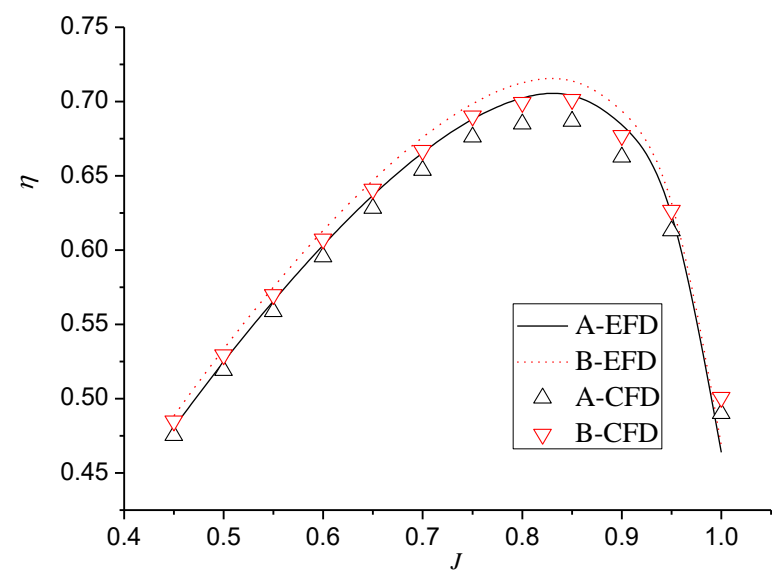

(c) Propulsive efficiency curves.

Fig. 8 Hydrodynamic performance curves of propulsion systems.

\subsection{Lateral forces with and without PBCF}

The pressure fluctuation on the blade, fin and rudder surface can cause the vibration of the propeller shaft and the rudder shaft. By observing the fluctuation of lateral force $F_{z}$, the vibration reduction effect of PBCF is analyzed. The lateral force direction is shown in Fig.9.

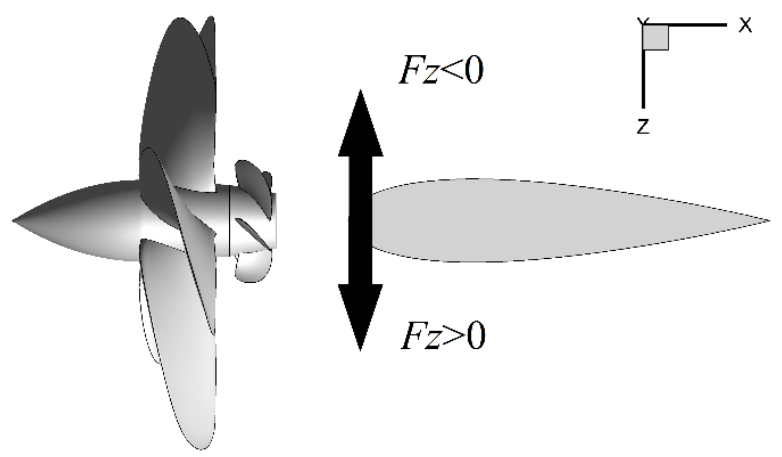

Fig. 9 Lateral force direction of propulsion system. 
Yu Sun, Tiecheng Wu, Yumin Su, Huanghua Peng
Numerical Prediction on Vibration and Noise Reduction Effects of Propeller Boss Cap Fins on a Propulsion System

Lateral force fluctuations of the propulsion systems with and without PBCF $(J=0.8)$ are illustrated in Fig. 10. It is found that there is basically no influence of PBCF on the blade and hub parts. By contrast, a significant change of the lateral force fluctuation on fins and hub cap has taken place. With the PBCF installed, the fluctuation amplitude of the hub cap is reduced, and the force period on the hub and fins becomes short. This weakened fluctuation intensity is beneficial to the vibration reduction of the propeller shaft. Meanwhile, due to the asymmetry along the rudder shaft, the rudder lateral force of the upper half is greater than the lower half. Therefore, there is a nonnegligible resultant force in the $Z$ direction. In many cases, the ship's course will be maintained by adjusting the rudder angle during the voyage, and it will cause partial sacrifice of the propulsion efficiency. After the installation of PBCF, there is no obvious change of the lateral force fluctuation, but the average force reduces from $-9.7 \mathrm{~N}$ to $-8.2 \mathrm{~N}$. With the lateral force reduced by $15.5 \%$.

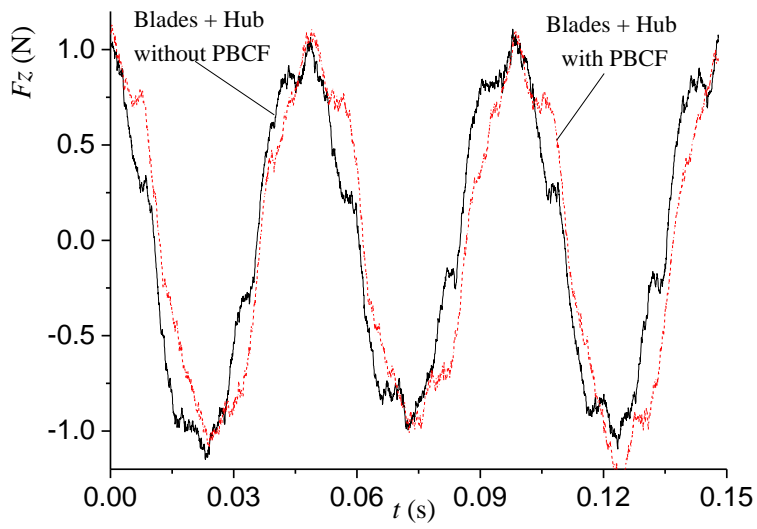

(a) Blades + Hub

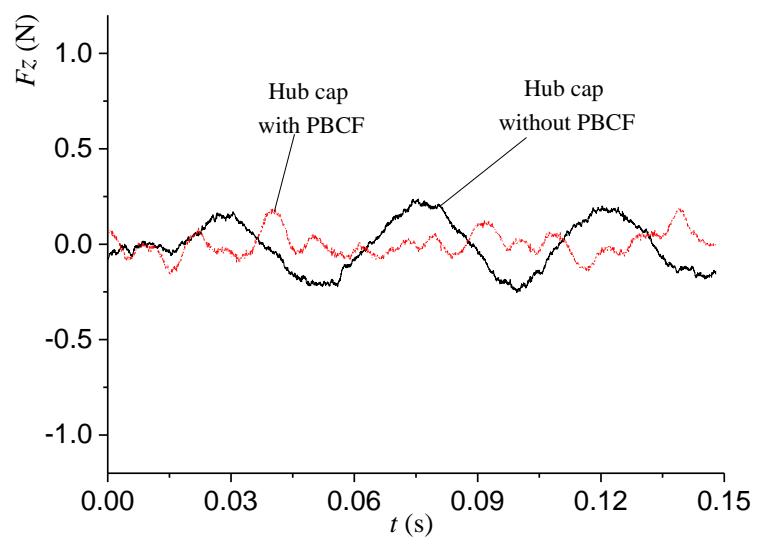

(b) Fins + Hub cap

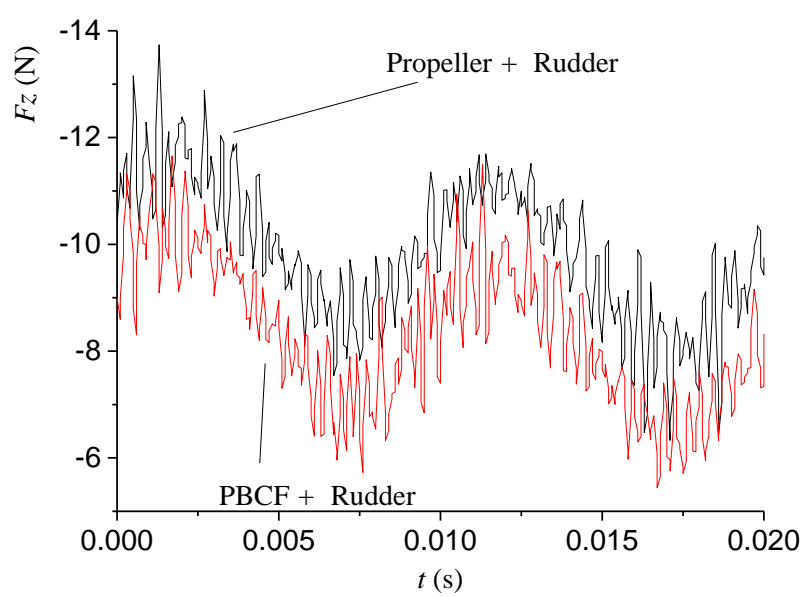

(c) Rudder

Fig. 10 Lateral force fluctuation with and without $\operatorname{PBCF}(J=0.8)$.

\subsection{Noise reduction effect analysis of PBCF}

After the contrast result of hydrodynamic performance obtained, the noise performance $(J=0.8)$ of the two systems is contrasted. The SPL of different angles at $r=10 \mathrm{~m}$ as shown in Fig. 11 is monitored, which are in the horizontal plane passing through the origin of the propeller disk. The consequence of noise performance is given in Fig. 12 and Table 3. In the following results, $0^{\circ}$ and $180^{\circ}$ are located in the downstream and upstream directions. 


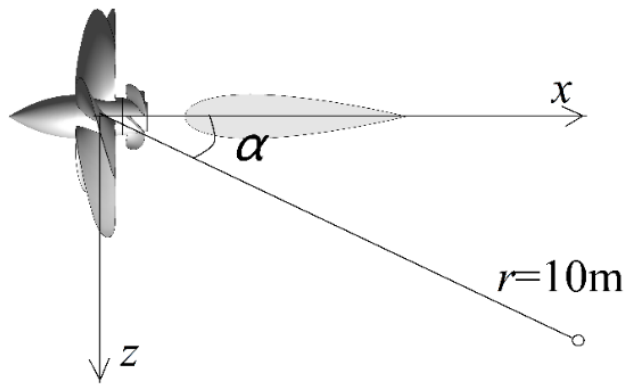

Fig. 11 Noise monitoring position of propulsion system.

Table 3 SPL of propulsion systems with and without PBCF $(J=0.8)$

\begin{tabular}{crrc}
\hline \hline \multirow{2}{*}{ Angle $\alpha\left(^{\circ}\right)$} & \multicolumn{2}{c}{ SPL $(\mathrm{dB})$} & $\begin{array}{c}\text { Noise Reduction } \\
(\mathrm{dB})\end{array}$ \\
\hline 0 & Without PBCF & With PBCF & 1.51 \\
45 & 127.10 & 125.59 & 1.41 \\
90 & 126.32 & 124.91 & 1.07 \\
135 & 124.79 & 123.71 & 1.33 \\
180 & 125.96 & 124.63 & 1.70 \\
225 & 126.66 & 124.95 & 1.40 \\
270 & 126.06 & 124.66 & 1.02 \\
315 & 124.63 & 123.61 & 1.35 \\
360 & 126.10 & 124.75 & 1.51 \\
\hline
\end{tabular}

The contrast results indicate that the SPL in the axial direction is about $2.2 \mathrm{~dB}$ higher than that in the radial direction. Accordingly, the noise reduction effect is also directional. The noise reduction effect in the axial direction is more significant, where the SPL can be reduced by more $0.56 \mathrm{~dB}$. The SPL spectrums $(J=0.8)$ in the axial and radial directions are displayed in Fig. 12 .

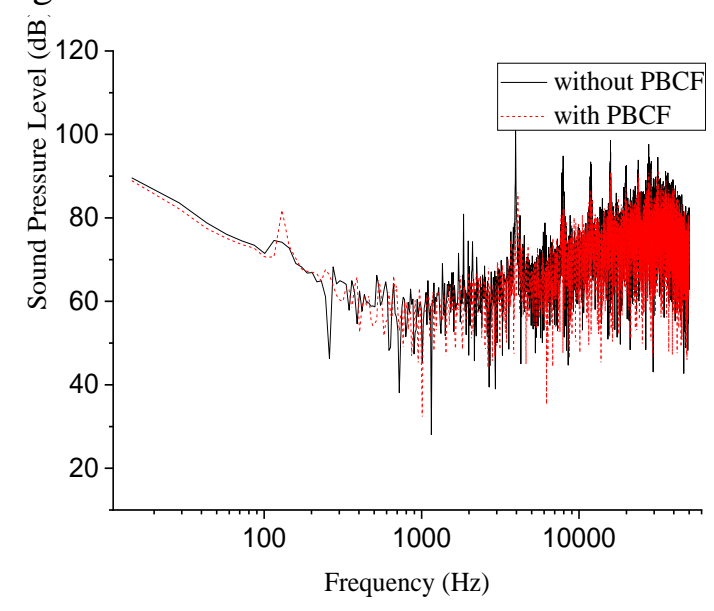

(a) Axial direction

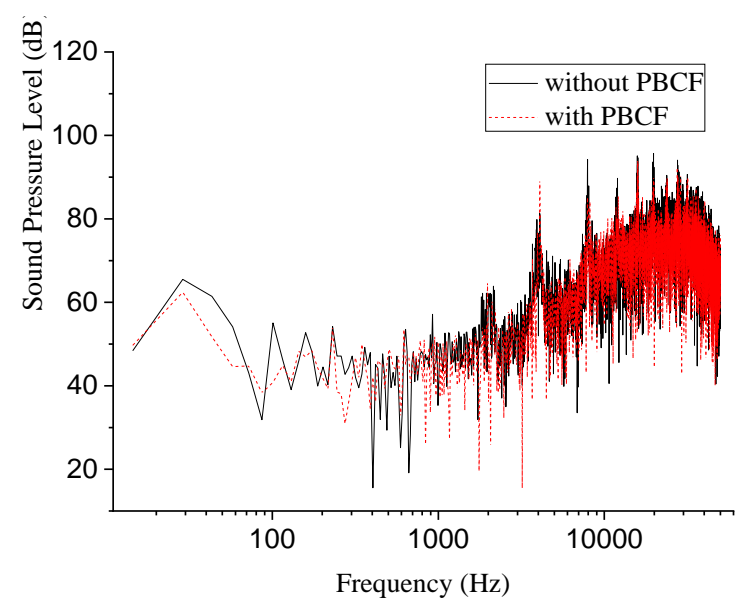

(b) Radial direction.

Fig. 12 SPL spectrums of propulsion systems $(J=0.8)$

The result of SPL spectrums shows that the SPL in the axial direction is as most as $20 \mathrm{~dB}$ greater than that in the radial direction in the frequency range of less than $1000 \mathrm{~Hz}$. Then the gap between them is gradually disappeared beyond that range. After the installation of PBCF, SPL of the propulsion system is reduced. While there is little impact of PBCF on the SPL peaks 
Yu Sun, Tiecheng $\mathrm{Wu}$, Yumin Su, Huanghua Peng
Numerical Prediction on Vibration and Noise Reduction Effects of Propeller Boss Cap Fins on a Propulsion System

at high frequencies, and the low frequency noise in the axial direction accounts for a larger proportion of the total SPL. Therefore, the noise reduction effect of PBCF in the axial direction is more obvious. In addition, the propeller wake flows around the rudder, and the accelerated rotation wake enhances its turbulent intensity. The changing flow field makes the SPL increased at high frequencies. The noise intensity of rudder becomes the major part of the total noise intensity at high frequencies. Therefore, the SPL spectrum of the propeller-rudder system is different from the propeller SPL spectrum.

\subsection{Flow field analysis}

The visual output of the CFD method is shown from Fig.13 to Fig.17. According to the flow field change of propulsion systems, the vibration and noise reduction mechanism of PBCF is analyzed in this section.
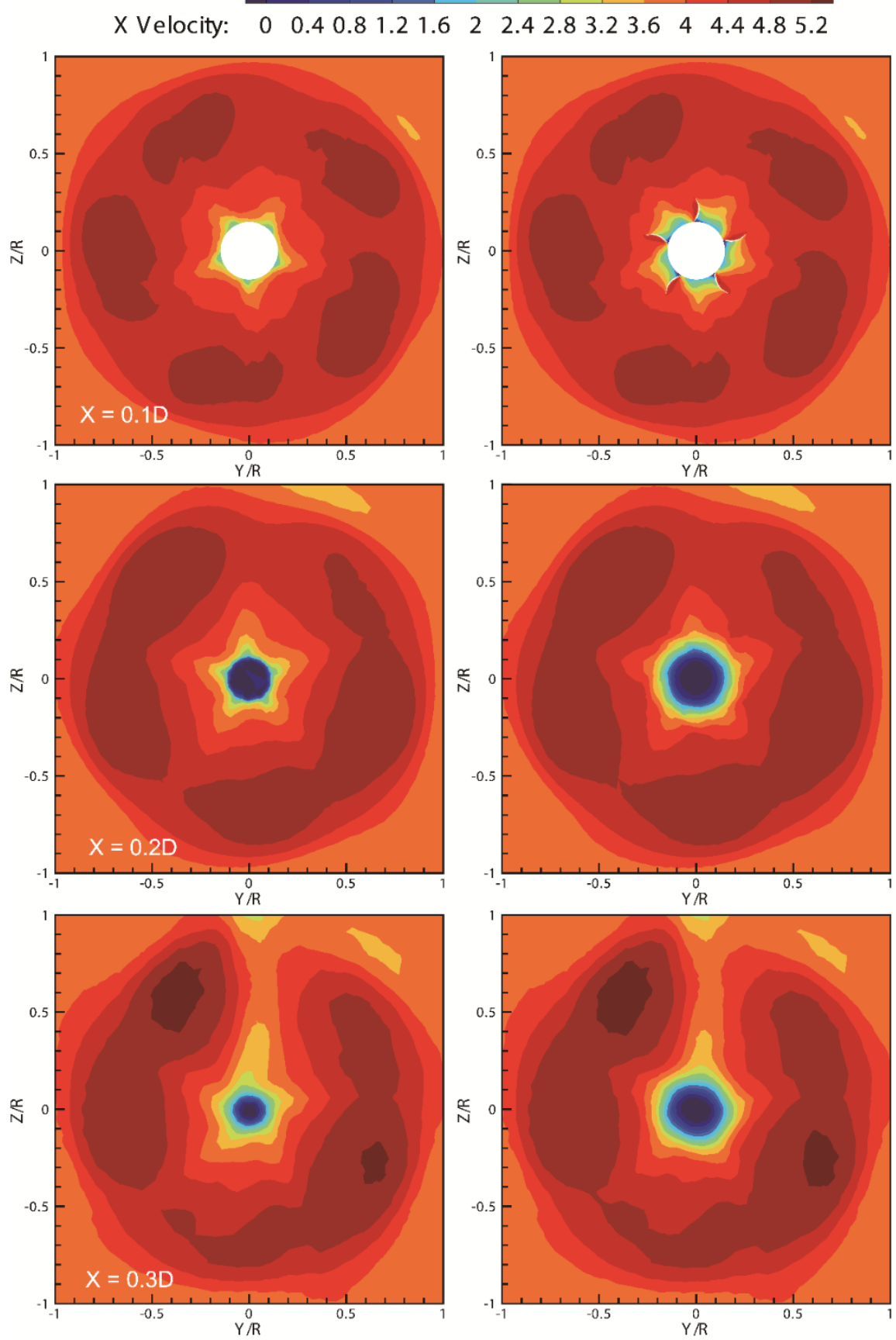
(a) Velocity distribution at different axial sections behind propeller (Left: Propeller + Rudder; Right: Propeller + $\mathrm{PBCF}+$ Rudder)

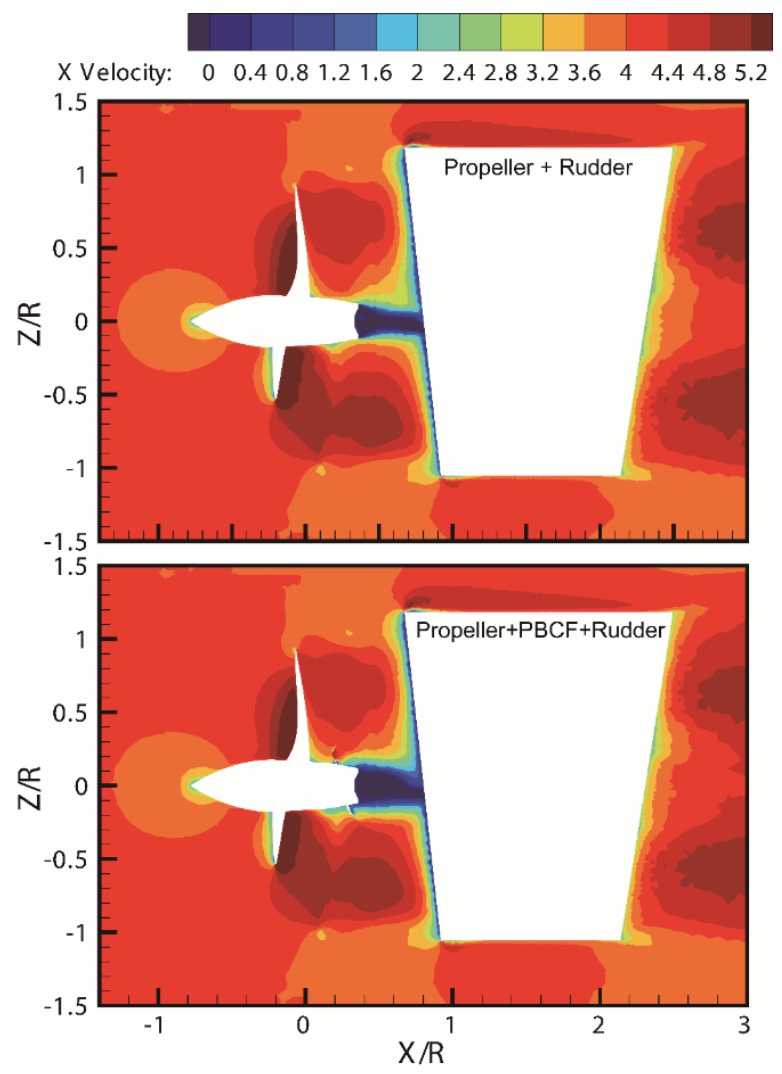

(b) Velocity distribution of $\mathrm{Y}=0$ tangent.

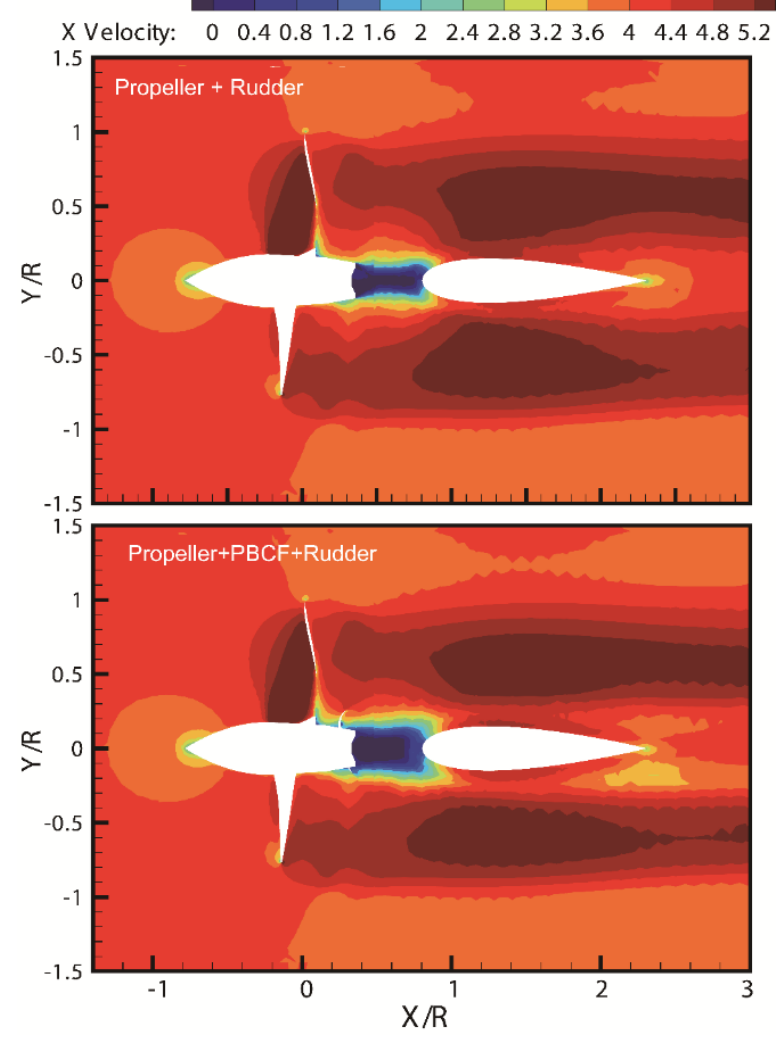

(c) Velocity distribution of $\mathrm{Z}=0$ tangent.

Fig. 13 Velocity distribution of propeller wake $(J=0.8)$.

The velocity distribution $(J=0.8)$ behind the propeller is illustrated in Fig. 13 , in which the contours show the axial velocity magnitudes. It is indicated that the velocity distribution at the centre of the section changes significantly. After the installation of PBCF, the axial velocity magnitude of the propeller wake increases. The magnitude of circumferential velocity behind PBCF reduces accordingly. The change of the flow field makes the energy of the hub vortex recycled. Therefore, the propulsive efficiency is enhanced. What's more, the wake velocity variation weakens the rudder lateral force magnitude due to the asymmetry and increases the course stability of a ship.

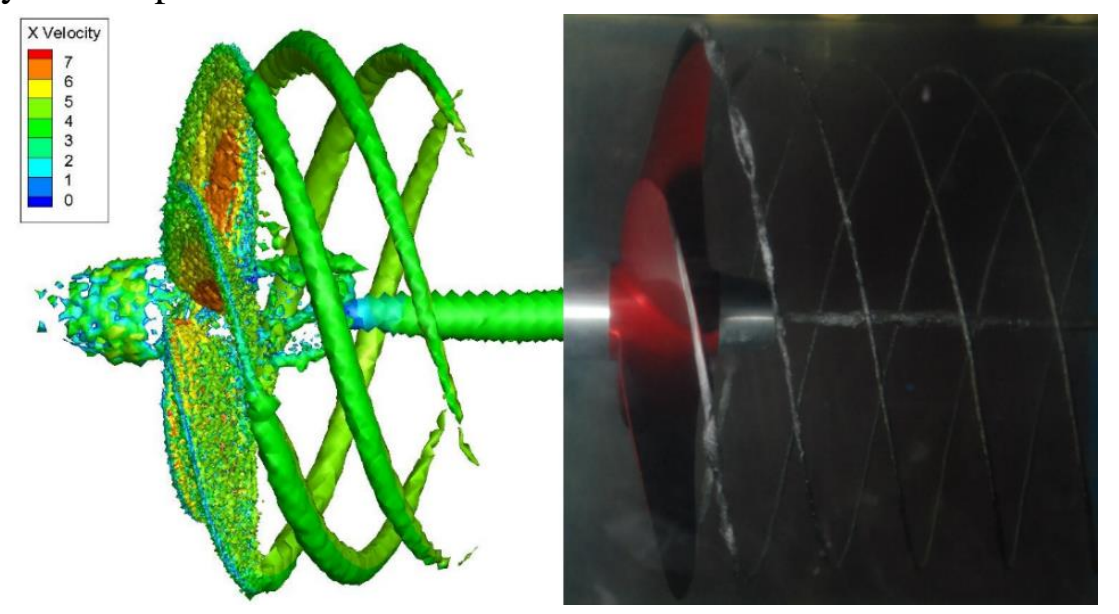

(a) Propeller. 
Yu Sun, Tiecheng Wu, Yumin Su, Huanghua Peng
Numerical Prediction on Vibration and Noise Reduction Effects of Propeller Boss Cap Fins on a Propulsion System

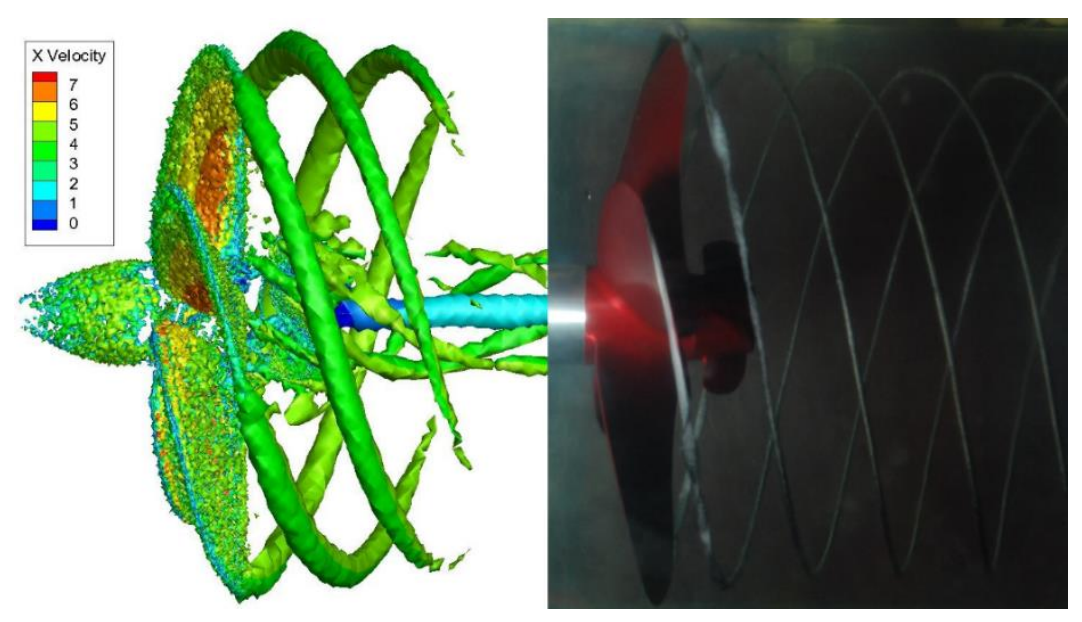

(b) Propeller + PBCF.

Fig. 14 Vortex structure without rudder $(J=0.8)$.

The vortex structures $(Q=5000)$ generated by the rotation of the propeller and PBCF are shown in Fig.14. In the tunnel, there is no cavitation at atmospheric pressure, and the cavitation grows by depressurizing the fluid pressure. The cavitation only represents the vortex intensity distribution in the tests. In the simulation cases, the vortex intensity at atmospheric pressure is analyzed. All the noises and pulsations are caused by the vortex intensity change. By contrast, it is found that the existence of PBCF has little effect on the tip vortex intensity of the propeller blades. Meanwhile the vortex behind the hub cap is reduced significantly, which makes the hub vortex weakened and accelerates the diffusion. In addition, a small amount of the tip vortex is generated by PBCF, but the influence on the propulsion system is not obvious. The fin tip vortex goes disappeared with the development of the wake.

In order to examine the flow field distribution of propulsion system, two planes are intercepted at different positions. The plane $\mathrm{A}(x=0.3 D)$ is located before the rudder, and the plane $\mathrm{B}(x=1.2 D)$ is located behind the rudder. The axial positions of the planes are given in Fig. 15.

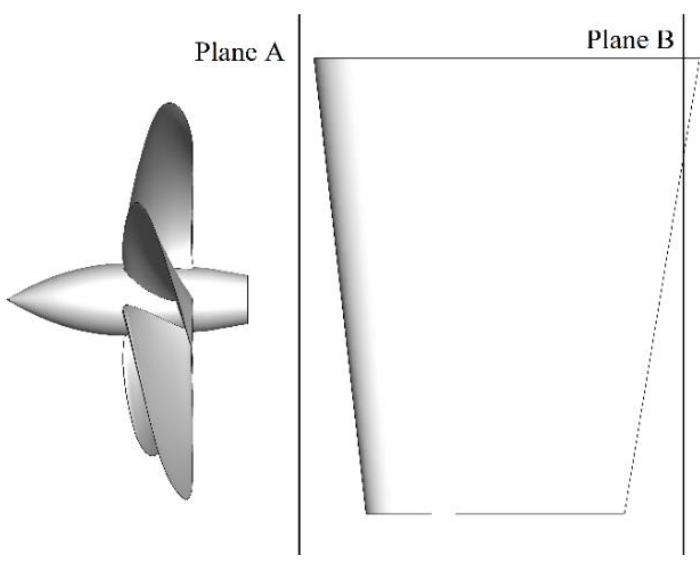

Fig. 15 Planes for comparison of vortex intensity distribution.

The vorticity distribution of the propulsion systems is shown in Fig. 16 and Fig.17. At the Plane A, the hub vortex intensity is reduced significantly by PBCF, and the vortex intensity of fin tips increases slightly. After the flow arriving at the Plane B, the contours of hub vortex expand and the intensity decreases, which means the vortex diffusion accelerated. The acoustic performance is related to the vortex distribution. The large-scale and small-scale vortex reductions result in the low and high frequency noise decreases, respectively. Thus, the flow wake change is coincided with the noise reduction effect of the PBCF. 


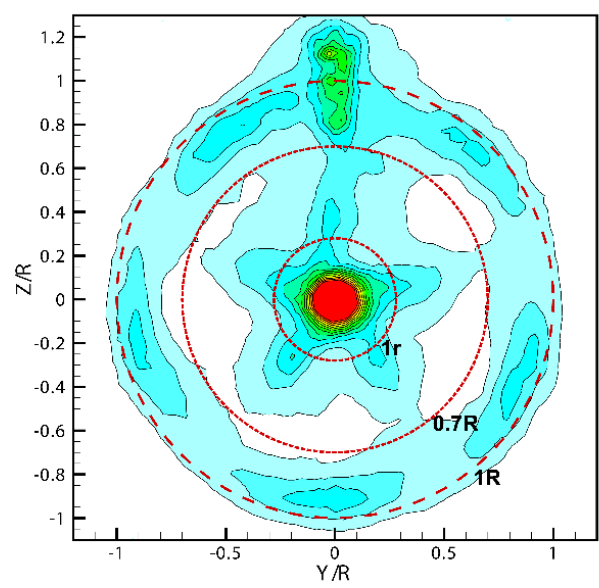

(a) Propeller + Rudder

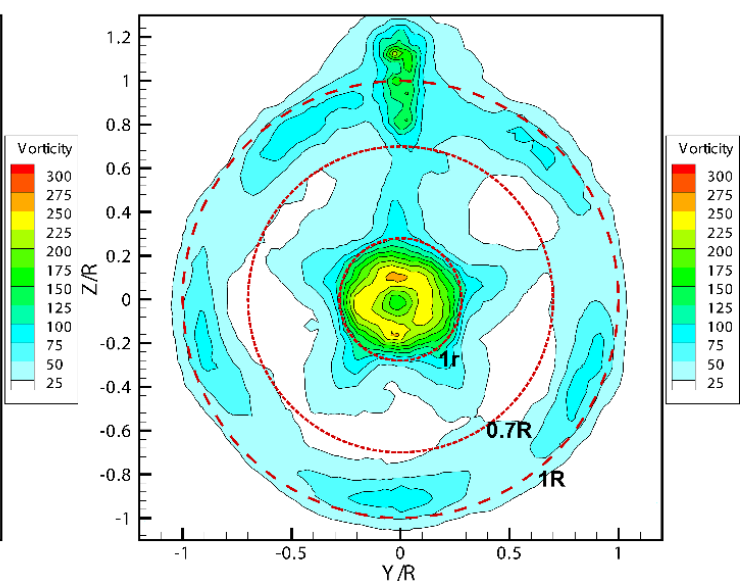

(b) Propeller + PBCF + Rudder

Fig. 16 Vortex intensity distribution at Plane A $(J=0.8)$.

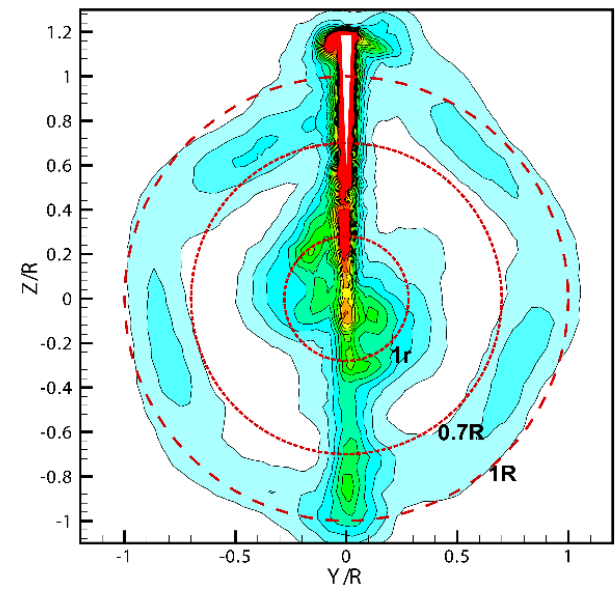

(a) Propeller + Rudder
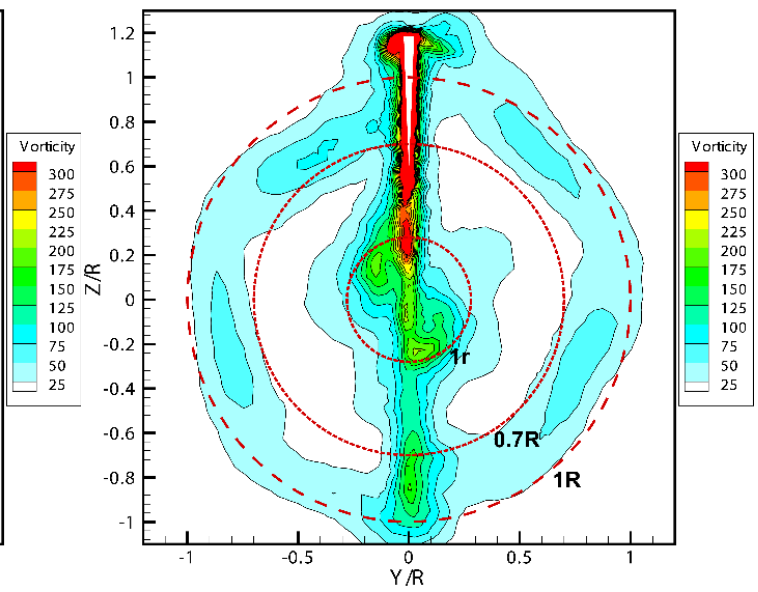

(b) Propeller + PBCF + Rudder

Fig. 17 Vortex intensity distribution at Plane B $(J=0.8)$.

\section{Conclusions}

The noise and vibration reduction effects of PBCF have been numerically investigated based on LES method and the FW-H equation. The numerical model is firstly verified by the grid convergence study, and further validated by the experimental data.

At the design advance coefficient $(J=0.8)$, the efficiency error is less than $3 \%$, which is within an acceptable range. Therefore, the numerical model is appropriate to evaluate the hydrodynamic performance of the propulsion systems. The calculation results show that the propulsion efficiency with PBCF is higher. The improvement of propulsive efficiency of PBCF is about $1.47 \%$ when $J=0.8$. In addition, the installation of PBCF can also reduce the lateral force fluctuation of the propeller shaft. By comparison, there is no significant change of the lateral force fluctuation amplitude of the rudder shaft, while the average value of lateral force is reduced by $15.5 \%$.

The installation of PBCF can reduce the hub vortex intensity, and this can help to accelerate its diffusion. The changes of the flow field make the SPL of the propulsion system reduced by as much as $1.51 \mathrm{~dB}$. What's more, the sound pressure level in the axial direction is about $2.2 \mathrm{~dB}$ higher than that in the radial direction. Accordingly, the noise reduction effect of PBCF is also directional, and the sound pressure level in the axial direction can be decreased by more $0.56 \mathrm{~dB}$. 
Yu Sun, Tiecheng $\mathrm{Wu}$, Yumin Su, Huanghua Peng
Numerical Prediction on Vibration and Noise Reduction Effects of Propeller Boss Cap Fins on a Propulsion System

Overall, it can be seen that PBCF is more than an energy saving device. Its function of noise reduction should be also very attractive. Since it is difficult to design and conduct such an experiment to measure the noise level, only the hydrodynamic performance experiment has been carried out in the cavitation tunnel. The future work may focus on how to design a scheme of the noise performance measurement.

\section{Acknowledgments}

This work was supported by the National Natural Science Foundation of China [Grant number 551009038], the Guangdong Basic and Applied Basic Research Foundation [Grant No. 2019A1515110721], the China Postdoctoral Science Foundation (Grant No. 2019M663243; 2013M540271) and the Fundamental Research Funds for the Central Universities [Grant No. NO.20 lgpy52].

\section{REFERENCES}

[1] Ouchi, K., Ogura, M., Kono, Y.. PBCF (Propeller Boss Cap Fins). Journal of the Society of Naval Architects of Japan, 1988, 163(6). https://doi.org/10.2534/jjasnaoe1968.1988.66

[2] Ouchi, K., Tamashima, M., Kawasaki, T., Koizuka, H.. A research and development of propeller boss cap fins (PBCF): 2nd report: study on propeller slipstream and actual ship performance. Journal of the Society of Naval Architects of Japan, 1989, 165, 43-53 (in Japanese). https://doi.org/10.2534/jjasnaoe1968.1989.43

[3] Ouchi, K., Tamashima, M.. Research and development of PBCF (propeller boss cap fin), new and practical device to enhance propeller efficiency. In: Proceedings of the 4th International Symposium on Practical Design of Ships and Mobile Units (PRADS), Bulgaria, 1989.

[4] Ouchi, K., Tamashima, M., Arai, K.. Propeller noise reduction caused by PBCF (propeller boss cap fins). Noise Control,1992. https://doi.org/10.5988/jime1966.27.768

[5] Dang, J.. An Exploratory Study on the Working Principles of Energy Saving Devices (ESDs): PIV, CFD Investigations and ESD Design Guidelines. Asme International Conference on Ocean, 2012, 8 (5) :12801287. https://doi.org/10.1115/OMAE2012-83053

[6] Hans, R. H., Tom, D. P., Nojiri, T., Model and Full Scale Evaluation of a 'Propeller Boss Cap Fins' Device Fitted to an Aframax Tanker. In: Second International Symposium on Marine Propulsors, smp'11, Hamburg, Germany, 2011.

[7] Kawamura, T., Ouchi, K., Nojiri, T.. Model and full scale CFD analysis of propeller boss cap fins (PBCF). Journal of Marine Science \& Technology, 2012, 17(4), 469-480. https://doi.org/10.1007/s00773-012-01812

[8] Vlašić, D., Degiuli, N., Farkas, A., Martić, I.. The preliminary design of a screw propeller by means of computational fluid dynamics. Brodogradnja: Teorija i praksa brodogradnje i pomorske tehnike, 2018, 69(3): 129-147. https://doi.org/10.21278/brod69308

[9] Lee, S., Paik, K. J.. URANS simulation of a partially submerged propeller operating under the bollard condition. Brodogradnja: Teorija i praksa brodogradnje i pomorske tehnike, 2018, 69(1): 107-121. https://doi.org/10.21278/brod69107

[10] Jang, Y. H., Eom, M. J., Paik, K. J., Kim, S. H., \& Song, G.. A numerical study on the open water performance of a propeller with sinusoidal pitch motion. Brodogradnja: Teorija i praksa brodogradnje i pomorske tehnike, 2020, 71(1): 71-83. https://doi.org/10.21278/brod71105

[11] Ghassemi, H., Mardan, A., Ardeshir, A..Numerical Analysis of Hub Effect on Hydrodynamic Performance of Propellers with Inclusion of PBCF to Equalize the Induced Velocity. Polish Maritime Research, 2012, 19(2), 17-24. https://doi.org/10.2478/v10012-012-0010-x

[12] Xiong, Y., Wang, Z., Qi, W.. Numerical Study on the Influence of Boss Cap Fins on Efficiency of Controllable-pitch Propeller. Journal of marine science and technology, 2013, 12(1), 13-20. https://doi.org/10.1007/s11804-013-1166-9

[13] Ma, C., Cai, H. P., Qian, Z. F., Chen, K.. The design of propeller and propeller boss cap fins (PBCF) by an integrative method. Journal of Hydrodynamics, 2014, 26(4), 586-593. https://doi.org/10.1016/S10016058(14)60066-4 
[14] Mizzi, K., Demirel, Y. K., Banks, C., Turan, O., Kaklis, P., Atlar, M.. Design optimisation of Propeller Boss Cap Fins for enhanced propeller performance. Applied Ocean Research, 2017, 62, $210-222$. https://doi.org/10.1016/j.apor.2016.12.006

[15] Gaggero, S. Design of PBCF energy saving devices using optimization strategies: A step towards a complete viscous design approach. Ocean Engineering, 2018, 159: 517-538. https://doi.org/10.1016/j.oceaneng.2018.01.003

[16] Wang, L. Z., Guo, C. Y., Su, Y. M., Xu P., Wu, T. C.. Numerical analysis of a propeller during heave motion in cavitating flow. Applied Ocean Research, 2017, 66: 131-145. https://doi.org/10.1016/j.apor.2017.05.001

[17] Wang, L. Z., Guo, C. Y., Xu P., Su, Y. M., Analysis of the performance of an oscillating propeller in cavitating flow, Ocean Engineering, 2018, 164: 23-39. https://doi.org/10.1016/j.oceaneng.2018.06.036

[18] Wang, L. Z., Martin, J. Z., Felli, M., Carrica, P. M., Experiments and CFD for the propeller wake of a generic submarine operating near the surface, Ocean Engineering, 2020, 206: 107304. https://doi.org/10.1016/j.oceaneng.2020.107304

[19] Bagheri, M. R., Mehdigholi, H., Seif, M. S., Yaakob, O.. An experimental and numerical prediction of marine propeller noise under cavitating and non-cavitating conditions. Brodogradnja: Teorija i praksa brodogradnje i pomorske tehnike, 2015, 66(2): 29-45.

[20] Aktas, B., Atlar, M., Fitzsimmons, P., Shi, W. C.. An advanced joint time-frequency analysis procedure to study cavitation-induced noise by using standard series propeller data. Ocean Engineering, 2018, 170: 329350. https://doi.org/10.1016/j.oceaneng.2018.10.026

[21] Wu, Q., Huang, B., Wang, G. Y., Cao, S. L., Zhu, M. M.. Numerical modelling of unsteady cavitation and induced noise around a marine propeller. Ocean Engineering, 2018, 160: 143-155. https://doi.org/10.1016/j.oceaneng.2018.04.028

[22] Ebrahimi, A., Razaghian, A. H., Seif, M. S., Zahedi, F., Nouri-Borujerdi, A.. A comprehensive study on noise reduction methods of marine propellers and design procedures. Applied Acoustics, 2019, 150: 55-69. https://doi.org/10.1016/j.apacoust.2018.12.004

[23] Cianferra, M., Petronio, A., Armenio, V.. Non-linear noise from a ship propeller in open sea condition. Ocean Engineering, 2019, 191: 106474. https://doi.org/10.1016/j.oceaneng.2019.106474

[24] Lilly, D. K.. Representation of small scale turbulence in numerical simulation experiments. Proceedings of IBM Scientific Computing Symposium on Environmental Sciences, 1967, 195-210.

[25] Mcmillan, O. J., Ferziger, J. H.. Direct testing of subgrid-scale models. Aiaa Journal, 1978, 1(12): 13401346. https://doi.org/10.2514/3.61313

[26] Clark, R. A.. Evaluation of subgrid-scale models using an accurately simulated turbulent flow. Journal of Fluid Mechanics, 1979, 91(3): 1-16. https://doi.org/10.1017/S002211207900001X

[27] Bhushan, S., Alam, M. F., Walters, D. K.. Evaluation of hybrid RANS/LES models for prediction of flow around surface combatant and Suboff geometries. Computers \& Fluids, 2013, 88(12), 834-849. https://doi.org/10.1016/j.compfluid.2013.07.020

[28] Wang, S. H., Shi, B. j., Li, Y. H., He, G. W.. A large eddy simulation of flows around an underwater vehicle model using an immersed boundary method. Theoretical and Applied Mechanics Letters, 2016, 6(6), 302305. https://doi.org/10.1016/j.taml.2016.11.004

[29] Posa, A., Balaras, E.. Large-Eddy Simulations of a notional submarine in towed and self-propelled configurations. Computers \& Fluids, 2018, 165, 116-126. https://doi.org/10.1016/j.compfluid.2018.01.013

[30] Wei, Q., Chen, H. X., Ma, Z.. A hybrid RANS/LES model for simulation of complex turbulent flow. Journal of Hydrodynamics, 2016, 28(5), 811-820. https://doi.org/10.1016/S1001-6058(16)60684-4

[31] Wang, L. Z., Guo, C. Y., Su, Y. M., Wu, T. C.. A numerical study on the correlation between the evolution of propeller trailing vortex wake and skew of propellers. International Journal of Naval Architecture \& Ocean Engineering, 2018, 10(2), 212-224. https://doi.org/10.1016/j.ijnaoe.2017.07.001

[32] Kim, H. J., Lee, S., Fujisawa, N.. Computation of unsteady flow and aerodynamic noise of naca0018 airfoil using large-eddy simulation. International Journal of Heat \& Fluid Flow, 2006, 27(2), 229-242. https://doi.org/10.1016/j.ijheatfluidflow.2005.08.007

[33] Li, D. Q., Hallander, J., Johansson, T.. Predicting underwater radiated noise of a full scale ship with model testing and numerical methods. Ocean Engineering, 2018, 161, 121-135. https://doi.org/10.1016/j.oceaneng.2018.03.027

[34] Kowalczyk, S., Felicjancik, J.. Numerical and experimental propeller noise investigations. Ocean Engineering, 2016, 120, 108-115. https://doi.org/10.1016/j.oceaneng.2016.01.032 
Yu Sun, Tiecheng $\mathrm{Wu}$, Yumin Su, Huanghua Peng
Numerical Prediction on Vibration and Noise Reduction Effects of Propeller Boss Cap Fins on a Propulsion System

[35] Rumpfkeil, M. P.. Using steady flow analysis for noise predictions. Computers \& Fluids, 2017, 154, 347357. https://doi.org/10.1016/j.compfluid.2017.03.003

[36] Asnaghi, A., Svennbergb, U., Bensowa, R. E.. Numerical and experimental analysis of cavitation inception behaviour for high-skewed low-noise propellers. Applied Ocean Research, 2018, 79, 197-214. https://doi.org/10.1016/j.apor.2018.07.010

[37] Wang, L. Z., Guo, C. Y., Xu P., Su, Y. M., Analysis of the wake dynamics of a propeller operating before a rudder, Ocean Engineering, 2019, 188: 106250. https://doi.org/10.1016/j.oceaneng.2019.106250

[38] Jiang, M., Li, X., Bai, B., Lin, D.. Numerical simulation on the naca0018 airfoil self-noise generation. Theoretical and Applied Mechanics Letters, 2012, 2(5), 052004. https://doi.org/10.1063/2.1205204

Submitted: $\quad 20.12 .2019$.

Accepted: $\quad$ 12.10.2020.
${ }^{a}$ Yu Sun,

b cTiecheng $\mathrm{Wu}^{*}$

wutch7@mail.sysu.edu.cn

dYumin $\mathrm{Su}$

${ }^{a}$ Huanghuang Peng

a College of Ocean Science and Engineering, Shanghai Maritime University, Shanghai 201306, China

b School of Marine Engineering and Technology, Sun Yat-sen University, Zhuhai 519000, China c Southern Marine Science and Engineering Guangdong Laboratory (Zhuhai), Zhuhai, 519000, China

d College of Shipbuilding Engineering, Harbin Engineering University, Harbin, 150001, China 